\title{
Peritoneal dissemination of recurrent small intestine primary GIST, successfully diagnosed by diffusion- weighted imaging
}

\author{
Junichiro Tanaka ${ }^{*}$, Katsuyuki Nakanishi ${ }^{1}$, Katsuhito Takahashi ${ }^{2}$ and Noriyuki Tomiyama ${ }^{3}$ \\ ${ }^{1}$ Department of Diagnostic \& Interventional Radiology, Osaka International Cancer Institute, Japan \\ ${ }^{2}$ Sarcoma Center, International University of Health and Welfare Mita Hospital, Japan \\ ${ }^{3}$ Department of Diagnostic \& Interventional Radiology, Osaka University Graduate School of Medicine, Japan
}

\begin{abstract}
An 81-year-old woman had undergone small intestine gastrointestinal stromal tumor (GIST) resection. Three years later, peritoneal dissemination appeared and was resected. She received imatinib therapy and was observed carefully. Three years after the first recurrence, diffusion-weighed imaging (DWI) of MRI revealed high intensity nodules on mesenterium. These nodules lacked $\left[{ }^{18} \mathrm{~F}\right]$-fluorodeoxyglucose $\left({ }^{18} \mathrm{~F}\right.$-FDG) uptake, but apparently increased, compared with DWI demonstrated a year before. Open biopsy was performed and many disseminated nodules were found on great omentum and mesenterium. Two of these nodules were resected and confirmed as peritoneal dissemination of GIST having mutation with at exon 9 of the c-kit gene. The expression of glucose transporter-1 (GLUT-1) was low level in our cases.
\end{abstract}

\section{Introduction}

Gastrointestinal stromal tumor (GIST) represents the most common mesenchymal tumor arising from the gastrointestinal tract. GISTs are most frequently found in the stomach $(60 \%)$, in the small bowel (30\%), and $10 \%$ are found elsewhere [1].

For patients, whose primary, localized GIST were resected, it is important to evaluate not only local recurrence, but also metastases and peritoneal dissemination. Contrast enhanced computed tomography (CT) is widely used because of the fast throughput of examinations and lower cost. Magnetic resonance imaging (MRI) is used supplementary with CT. Fused $\left[{ }^{18} \mathrm{~F}\right]$-fluorodeoxyglucose positron emission tomography (PET)/CT is used to clarify if CT or MRI is ambiguous, but it is useless for GIST without ${ }^{18} \mathrm{~F}-\mathrm{FDG}$ uptake [2-4]. On the other hand, diffusion-weighed imaging (DWI) is becoming important in the assessment of malignant tumors [5]. We present a case of recurrent small intestine primary GIST, which was successfully diagnosed by DWI.

\section{Case report}

An 81-year-old woman underwent small intestine gastrointestinal stromal tumor resection. Three years later, peritoneal dissemination appeared and was resected. Since then she was administered imatinib and was observed carefully with CT, MRI with DWI and ${ }^{18} \mathrm{~F}-\mathrm{FDG}$ PET/CT.

Three years after the first recurrence, MRI was performed and DWI revealed high intensity nodules on the mesenterium. Compared with DWI of the previous year, theses high intensity nodules became apparently more obvious and they were considered to be recurrences (Figure 1). However, on PET/CT they did not have ${ }^{18} \mathrm{~F}-\mathrm{FDG}$ uptake (Figure 2). Open biopsy was performed and many disseminated nodules were found on great omentum and mesenterium. Two of these nodules were resected and confirmed as peritoneal dissemination of GIST having mutation with at exon 9 of the c-kit gene. The expression of glucose transporter-1 (GLUT-1) was low level in our cases (Figure 3).

We got the diagnosis of recurrent GIST. Consequently the treatment was immediately changed to sunitinib therapy, which is more effective for GIST having mutation at exon 9 of the $c$-kit gene. The patient is undergoing sunitinib therapy.

\section{Discussion}

The standard imaging technique in patients with GIST should be contrast- enhanced CT [3]. Contrast-enhanced CT is widely used, because of the fast throughput of examinations and lower cost. MRI has been used supplementarily with contrast-enhanced CT [2,3]. Recently, PET/CT is playing an important role in the assessment of malignant tumors. In particular, PET/CT imaging can be considered a reliable tool for assessment of GIST response to targeted therapy such as imatinib [5]. However, Choi et al. reported that $21 \%$ of lesions did not show appreciable glucose uptake on pretreatment PET [4]. In such cases, PET is useless and a different imaging modality may be required. In our case, on pathological evaluation, the expression of GLUT-1 was low level and it indicated that these recurrent nodules lacked ${ }^{18} \mathrm{~F}-\mathrm{FDG}$ uptake.

Correspondence to: Junichiro Tanaka, Department of Diagnostic \& Interventional Radiology, Osaka International Cancer Institute, 3-1-69, Otemae, Chuo-ku, Osaka, zip 541-8567, Japan, Tel: +81 66945 1181; Fax: +81-6-6945-1883; E-mail: tanaka_j@d2.dion.ne.jp

Key words: peritoneum, MR-imaging, treatment effects

Received: December 13, 2017; Accepted: December 27, 2017; Published: December 29, 2017 

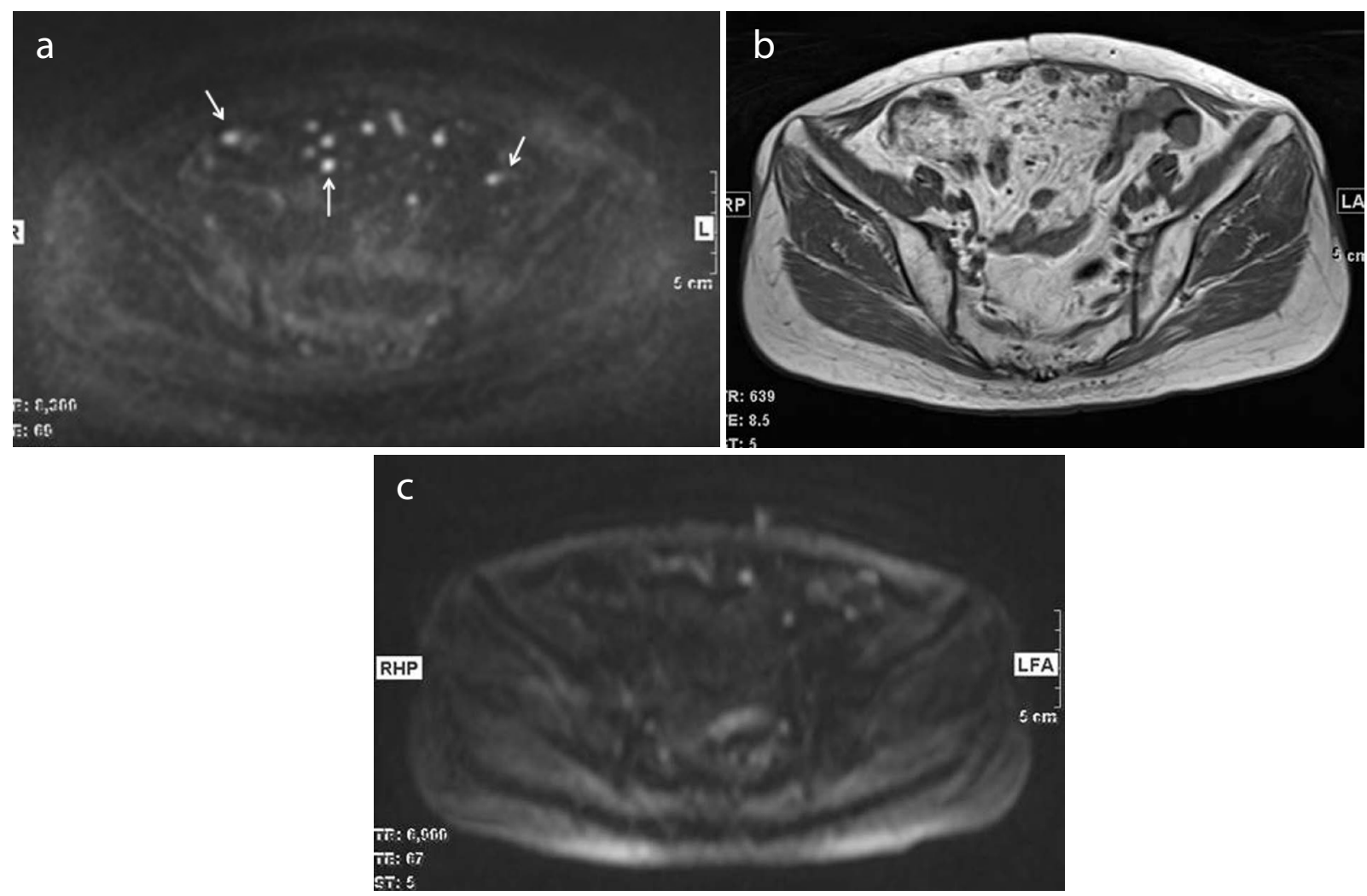

Figure 1. (a) Axial diffusion-weighted image (DWI) (b factor=1000 s/mm2) shows high intensity nodules on the mesenterium (white arrows). (b) Some nodules are detected on axial T1-weighted image but these were fuzzy.

(c) Axial DWI a year before showed high intensity nodules but they are fewer compared with (a).
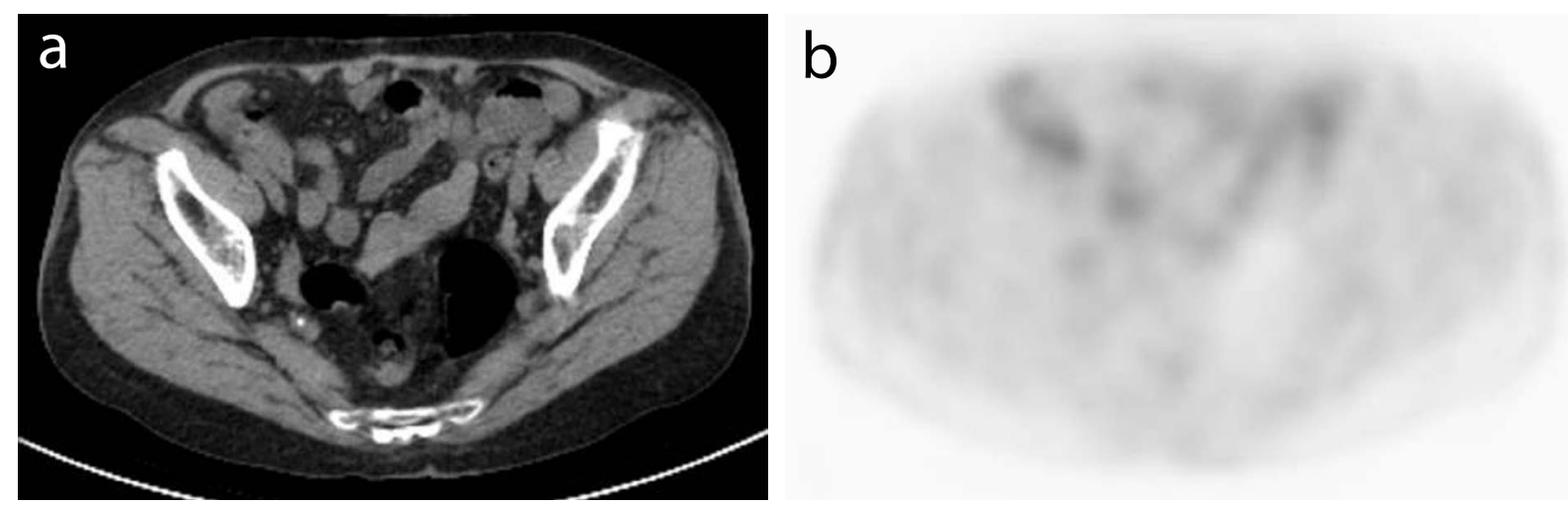

Figure 2. PET/CT image shows that some nodules are detected on CT (a) but they do not have abnormal FDG uptake on PET (b).

Diffusion-weighted imaging (DWI) has been more widely used in oncology patients because of its noninvasiveness and it is becoming important in the evaluation of malignant tumors [6,7]. Malignant tumors are frequently more cellular than the tissue from which they originate and thus appear to be of relatively high signal intensity at DWI, because of restriction of water movement [7]. Some radiologists compared the accuracy of MRI with DWI and PET/CT for diagnosing peritoneal dissemination [6-9]. Satoh et al. compared the diagnostic performance of PET/CT, MRI with and without DWI, and contrast enhanced MDCT in the detection of peritoneal dissemination of malignant tumors. They concluded that PET/CT is the most useful and DWI can be used as a screening tool if PET/CT is not available [8]. On the other hand, Soussan et al. [9] reported that PET/CT and MR-DWI showed a similarly high accuracy in diagnosing peritoneal carcinomatosis from gastrointestinal malignancy. In our case, DWI was a useful method in detecting dissemination of recurrent GIST.

In summary, after the second operation we detected small nodules in the abdominal cavity on PET/CT but they lack ${ }^{18} \mathrm{~F}-\mathrm{FDG}$ uptake. It was difficult to determine if these are recurrences or not. Although, on DWI these nodules apparently increased, compared with the DWI from the previous year and they were considered to be recurrences. Open biopsy was performed and we got the diagnosis of recurrent GIST. MRI with DWI may be a useful follow-up method for GIST without ${ }^{18} \mathrm{~F}$-FDG uptake after surgery.

\section{Acknowledgement}

We are indebted to Hisako Yamamura for pathological diagnosis of GIST. 

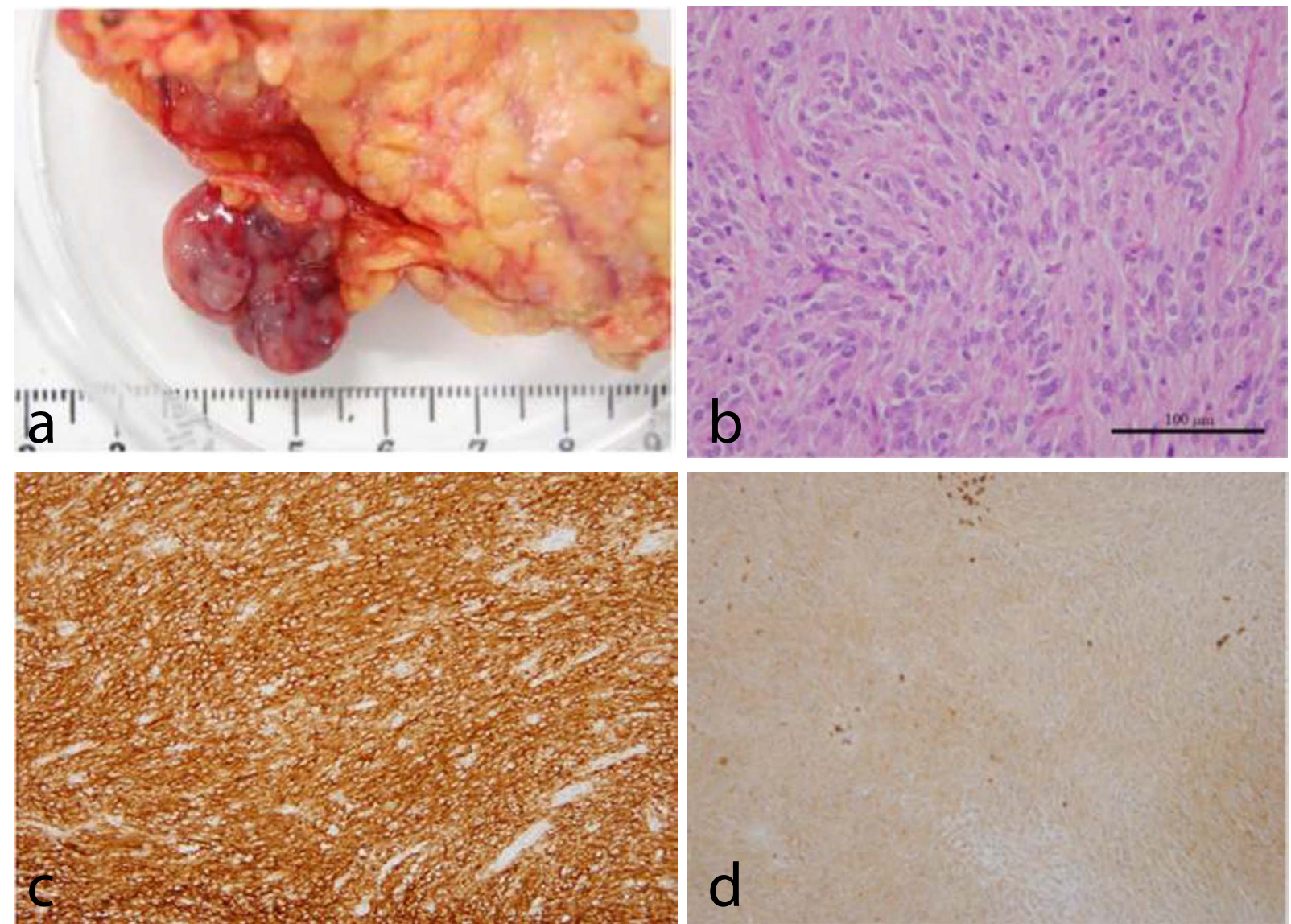

Figure 3. (a) The photograph of one resected nodule of peritoneal dissemination.

(b) Hematoxylin-and-eosin staining shows that the tumor is composed of spindle-shaped cells.

(c) Immunohistochemical staining confirms KIT strongly positive GIST diagnosis.

(d) The expression of Glucose transporter-1 is lower, compared with those in our previous cases.

\section{References}

1. Miettinen M, Sarlomo-Rikala M, Lasota J (1999) Gastrointestinal stromal tumors: recent advances in understanding of their biology. Hum Pathol 30: 1213-1220. [Crossref]

2. NCCN guidelines (2014) http://www.nccn.org/professionals/physician_gls/f_guidelines. asp.

3. Kalkmann J, Zeile M, Antoch G, Berger F, Diederich S, et al. (2012) Consensus report on the radiological management of patients with gastrointestinal stromal tumours (GIST): recommendations of the German GIST Imaging Working Group. Cancer Imaging 12: 126-135. [Crossref]

4. Choi H, Charnsangavej C, de Castro Faria S, Tamm EP, Benjamin RS, et al. (2004) CT evaluation of the response of gastrointestinal stromal tumors after imatinib mesylate treatment: a quantitative analysis correlated with FDG PET findings. AJR Am J Roentgenol 83: 1619-1628. [Crossref]

5. Antoch G, Kanja J, Bauer S, Kuehl H, Renzing-Koehler K, et al. (2004) Comparison of PET, CT, and dual-modality PET/CT imaging for monitoring of imatinib (STI571) therapy in patients with gastrointestinal stromal tumors. $J$ Nucl Med 45: 357-365. [Crossref]

6. Takahara T, Imai Y, Yamashita T, Yasuda S, Nasu S, Van Cauteren M (2004) Diffusion weighted whole body imaging with background body signal suppression (DWIBS) technical improvement using free breathing, STIR and high resolution 3D display. Radiat Med 22: 275-282. [Crossref]

7. Koh DM, Collins DJ (2007) Diffusion-weighted MRI in the body: applications and challenges in oncology. AJR Am J Roentgenol 188: 1622-1635. [Crossref]

8. Satoh Y, Ichikawa T, Motosugi U, Kimura K, Sou H, et al. (2011) Diagnosis of peritoneal dissemination: comparison of $18 \mathrm{~F}-\mathrm{FDG}$ PET/CT, diffusion-weighted MRI, and contrast-enhanced MDCT. AJR Am J Roentgenol 196: 447-453. [Crossref]

9. Soussan M, Des Guetz G, Barrau V, Aflalo-Hazan V, Pop G, et al. (2012) Comparison of FDG-PET/CT and MR with diffusion-weighted imaging for assessing peritoneal carcinomatosis from gastrointestinal malignancy. Eur Radiol 22: 1479-1487. [Crossref]

Copyright: (C2017 Tanaka J. This is an open-access article distributed under the terms of the Creative Commons Attribution License, which permits unrestricted use, distribution, and reproduction in any medium, provided the original author and source are credited. 